\title{
Kann die Schweiz von den Erfahrungen in anderen Ländern profitieren?
}

\section{Bernd Mühlbauer ${ }^{a}$ \\ Heinz Locher ${ }^{b}$ \\ a Prof. Dr. med. \\ b Dr. rer. pol.}

Korrespondenz:

Dr. rer. pol. Heinz Locher Postfach 266

CH-3000 Bern 15

heinz@locher-bern.ch

www.locher-bern.ch

Prof. Dr. med. Bernd Mühlbauer Direktor Institut für Pharmakologie

Klinikum Bremen Mitte

D-28177 Bremen

\section{muehlbauer@}

pharmakologie-bremen.de

www.pharmakologie-bremen.de
Tarife und Preise für medizinische Leistungen (niedergelassene Ärzte, Spitäler) werden in der Schweiz grundsätzlich in Verträgen zwischen Versicherern und Leistungserbringern vereinbart. Bei Medikamenten und zum Teil bei Medizinalprodukten (MiGeL) hingegen werden sie von der zuständigen Behörde als Höchstpreis oder Höchstvergütungsbetrag festgesetzt. Als Voraussetzung für eine Vergütung müssen die Leistungen die Kriterien der Wirksamkeit, der Zweckmässigkeit und der Wirtschaftlichkeit (WZW) erfüllen. Dem Preisüberwacher stehen dabei Mitwirkungsrechte zu.

\section{Vergütungssysteme im politischen Brennpunkt}

Die verschiedenen Vergütungssysteme, die Prozesse der Festlegung und die Höhe der Tarife und Preise stehen als Folge der Kostenentwicklung im Gesundheitswesen zunehmend im politischen Brennpunkt.
Eine stärkere Regelung erscheint aus einer marktwirtschaftlich-wettbewerblichen Sicht grundsätzlich unbefriedigend. Die Liste der zur Vergütung durch die OKP (Obligatorischen Krankenpflegeversicherung) zugelassenen Medikamente (Spezialitätenliste) samt deren Preisen, die MiGeL mit ihren Höchstvergütungsbeträgen und die Analysetarife gehören zudem zu den wenigen unmittelbaren Eingriffsmöglichkeiten des Bundesrates zur kurzfristigen Beeinflussung der von der OKP zu tragenden Kosten. Diese Kompetenzen nutzt er in letzter Zeit intensiver. Bezogen auf den Anteil der Medikamentenkosten und der MiGeL an den OKP-Gesamtkosten erscheint diese Regulierung als überproportional, was Kritiker eine Schwächung des Forschungs-, Pharma- und Medizinproduktestandorts Schweiz befürchten lässt.

Die Prozesse und Kriterien zur Festlegung der Preise und Höchstvergütungsbeträge sind regelmässig Gegenstand intensiver Auseinandersetzungen, bei-

\section{Symposium im Marriott Hotel, Zürich, 30. August 2011}

\section{Bewertung von medizinischen Leistungen und Preisbildung bei Medikamenten und Medizinprodukten}

Innovative Lösungsansätze, auch aus internationaler Sicht, für die Schweiz

Liste der Referenten

- Dr. iur. Thomas Heiniger, Regierungsrat und Gesundheitsdirektor des Kantons Zürich (Eröffnung)

- Dr. rer. pol. Heinz Locher, Management + Consulting Services, Bern (Einleitungsreferat)

Impuls-Referate

- A. Faller, lic. iur., Advokat, Vizedirektor und Leiter des Direktionsbereichs Kranken-, Unfall- und Militärversicherung des Bundesamtes für Gesundheit (BAG)

- Prof. Dr. Eberhard Wille, Lehrer für Volkswirtschaftslehre, Universität Mannheim, Vorsitzender des Gesundheits-Sachverständigenrates im Gesundheitsministerium, Berlin

- Dr. Gian Carlo Sciuchetti, Geschäftsführer «Markt und Erstattung» Verband der forschenden Pharmaunternehmen, Berlin

- Dr. Peter Huber, Geschäftsführer Intergenerika

- Dr. Melchior Buchs, Generalsekretär, Dachverband der Schweizerischen Handels- und Industrievereinigung der Medizintechnik FASMED

- Stefan Wild, External Affairs Director, MSD Schweiz

- Prof. Dr. med. Bernd Mühlbauer, Direktor des Instituts für Klinische Pharmakologie am Klinikum Bremen:

- Stefan Meierhans, Preisüberwacher - Eidgenössisches Volkswirtschaftsdepartement, Bern

- Dr. Christopher Hermann, Stellvertretender Vorstandsvorsitzender der AOK Baden-Württemberg

- Professor Dr. Thomas D. Szucs, Verwaltungsratspräsident der HELSANA, Krankenversicherer

- PD Dr. Marcel Mesnil, Generalsekretär, pharmaSuisse, Schweizerischer Apothekerverband

- Reinhard Kuster, Leiter Einkauf, Kantonsspital St. Gallen

- Dr. Jacques-Henri Weidmann, Director Public Affairs, Market Access and Communication, sanofi-aventis (suisse) sa

- Dr. pharm. Enea Martinelli, Chefapotheker, Spital Interlaken, Mitglied im Kantonsparlament

Die Veranstaltung dauert von 9 Uhr bis ca. 18 Uhr und umfasst neben den Referaten auch zwei Podiumsdiskussionen.

Detailliertes Programm und Anmeldung unter www.rsmedicalconsult.com

Die Schweizerische Ärztezeitung ist Medienpartner des Symposiums 


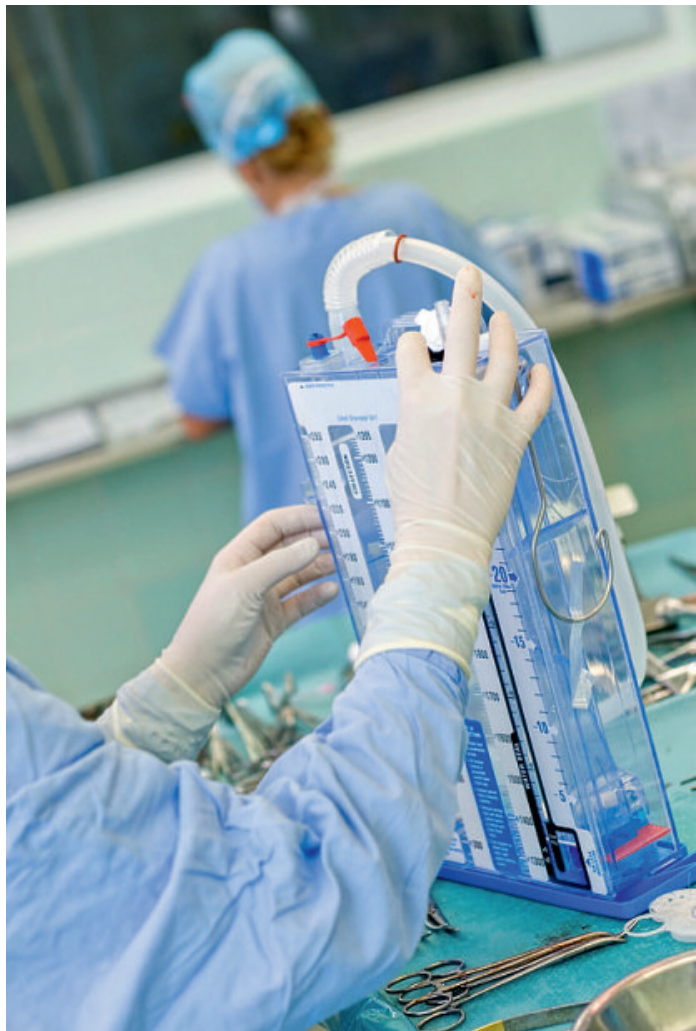

Der frühe Einsatz von Nutzenbewertungsinstrumenten soll die Unterscheidung echter Innovationen von solchen mit keinem oder marginalem Zusatznutzen für den Patienten erleichtern.

spielsweise bezüglich der in die verlangten Auslandspreisvergleiche einzubeziehenden Länder. Mit diesen Vergleichen werden in der Regel Behördenpreise mit ebensolchen verglichen, selbst wenn in Staaten mit entsprechenden Regulierungsmöglichkeiten die effektiv bezahlten Preise niedriger sind.

\section{Innovationen: Spreu vom Weizen trennen}

Ebenfalls kontrovers wird derzeit diskutiert, inwieweit die generellen WZW-Grundsätze des Krankenversicherungsgesetzes (KVG) - «wirksam, zweckmässig, wirtschaftlich» - und die Ergebnisse hierfür entwickelter Messinstrumente des Health Technology Assessments (HTA) in die Preisfindung einbezogen werden. Der frühe Einsatz von Nutzenbewertungsinstrumenten soll die Unterscheidung echter Innovationen von solchen mit keinem oder marginalem $\mathrm{Zu}$ satznutzen für den Patienten erleichtern, um ersteren eine rasche Zulassung und eine angemessene Tarifierung zu ermöglichen. In der Schweiz bestehen hier noch institutionelle Mängel, beispielsweise fehlt der systematische Zugang zu einer oder mehreren HTAAgenturen.

Die Preise der überwiegenden Zahl von Medizinprodukten, die nicht auf der MiGeL stehen, bilden sich zwar auf dem Markt, die damit verbundenen medizinischen Leistungen müssen aber ebenfalls die WZW-Voraussetzungen erfüllen. Auch hier bestehen die genannten Mängel bezüglich des Health Technology Assessments.

Eine vom gesetzlichen Zulassungswesen unabhängige wissenschaftliche Nutzenbewertung hat inzwischen eine über 15-jährige Tradition, wobei Gründungen von Institutionen wie des NICE oder des IQWiG im deutschsprachigen Raum als Meilensteine angesehen werden können (siehe Kasten unten). Auch hier haben diese Entwicklungen zum Teil heftige Diskurse ausgelöst. So ist kaum zu bezweifeln, dass die Schweiz von den einschlägigen Erfahrungen in Ländern mit einer systematischen Bewertung des patientenrelevanten Nutzens medizinischer Innovationen profitieren kann.

Das Symposium in Zürich zu diesem Thema soll den verschiedenen Beteiligten ermöglichen, unterschiedliche Sichtweisen und Erfahrungen auszutauschen.

\section{NICE und IQWiC}

Das National Institute for Health and Clinical Excellence (NICE) wurde 1999 in Grossbritannien als Sondergesundheitsbehörde des staatlichen Gesundheitsdienstes (National Health Service, NHS) für England und Wales gegründet. Aufgabe des NICE ist es, möglichst umfassende und verlässliche Empfehlungen für Patienten, die medizinische Fachwelt und die Öffentlichkeit zu den aktuellen «Best Practices» abzugeben.

In Deutschland wurde im Zuge der Gesundheitsreform vom 1. Juni 2004 das Institut für Qualität und Wirtschaftlichkeit im Gesundheitswesen (IQWiG) gegründet, welches als unabhängiges wissenschaftliches Institut im Auftrag des Gemeinsamen Bundesausschusses (GBA) und des Bundesgesundheitsministeriums (BMG) tätig ist. $\mathrm{Zu}$ den Aufgaben des Instituts gehören ähnlich wie beim NICE unter anderem die Bewertung von Operations- und Diagnoseverfahren, Arzneimitteln sowie Behandlungsleitlinien. Auf der Basis der evidenzbasierten Medizin erarbeitet das IQWiG ausserdem die Grundlagen für neue Disease-ManagementProgramme (DMP) - struk_ turierte Behandlungsprogramme für chronisch Kranke. 